\title{
Prosopometamorphasia: The Presenting Symptom in a Case of Schizophrenia
}

\author{
Dawn De Souza ${ }^{a, b}$
}

\begin{abstract}
Prosopometamorphosia is a rare visual perceptual distortion resulting in altered perception of faces. It is of unknown cause, but is usually attributed to organic diseases of neurological origin such as migraine, epilepsy and abnormal findings on CT scans and MRIs of the head. This case illustrates the presentation of a young female with prosopometamorphasia and discusses the diagnostic and medication challenges over the years before it resolved with clozapine.
\end{abstract}

Keywords: Prosopometamorphosia; Schizophrenia; Psychosis; Visual hallucinations; Face

\section{Introduction}

The term prosopometamorphopsia was introduced by the British neurologist Macdonald Crichley, to denote a type of metamorphopsia in which people's faces or specific parts of faces are perceived in a distorted manner [1]. Metamorphosias are typically associated with eye or brain diseases.

Slade and Bentall (1988) suggested that hallucinations occur as a result of the inability of the subject to distinguish if an object is real or if it is a product of the imagination, i.e. hallucinations would be caused by a deficit in the metacognitive capacity of assessment/discrimination of reality [2]. Illusions of distorted faces relate to activity on the lateral convexity of the occipital lobe 39, a region specialized for face features (the occipital face area), and the same region is likely to account for hallucinations of distorted faces [3].

Hallucinations and delusions are core diagnostic criteria for schizophrenia [4]; however, visual hallucinations and illusions are less common as the presenting symptom [5]. Psychiatrists are trained to ask about hallucinations; however, visual illusions and hallucinations are usually taken as an indication of organic

Manuscript submitted August 26, 2018, accepted September 4, 2018

${ }^{a}$ Department of Psychiatry, University of Saskatchewan, 103 Hospital Drive, Saskatoon SK S7N 0W8, Canada

${ }^{b}$ Corresponding Author: Dawn De Souza, Department of Psychiatry, University of Saskatchewan, 103 Hospital Drive, Saskatoon SK S7N 0W8, Canada. Email:dpd227@mail.usask.ca

doi: https://doi.org/10.14740/jmc3140w conditions given the commonality of auditory hallucinations [6]. Prosopometamorphosia [1] is even rarer, and is usually attributed to a neurological cause $[5,6]$. A search of PubMed, using the term prosopometamorphosia, lists 15 cases all linked to organic causes, with diagnoses of epilepsy, migraine, eye diseases, CT and MRI abnormalities [7-9], none of which were attributed to schizophrenia. Treatment approaches were variable and specific to those conditions. Prosopometamorphosia, as the presenting feature, is rarely attributed to a diagnosis of schizophrenia [10], although identified as a feature of schizophrenia [11]. Thus, it may present diagnostic delays and management challenges, with distress and high-risk behavior for the patient, sometimes taking years before resolving as in the case of Ms. AB.

\section{Case Report}

Ms. AB, a 22-year-old university student, was on holiday with her family at the time of presentation. The family noticed a sudden, marked deterioration where she refused to leave the hotel, and had picked up a knife to harm herself. On presentation, she reported that she saw people around her having distorted faces, "noses moving around", not attached to people's faces, and she covered her eyes in distress at one point, stating that the interviewer's eyes had changed to cat's eyes, and then that they were moving from side to side on the interviewer's face. She also reported seeing things as "fake" and "detached", like through a veil, and that she could not concentrate and had poor memory. She denied auditory, tactile, olfactory or other visual hallucinations at the time. No other delusions were reported at the time.

About 2 - 3 months later, she reported feeling like her facial expressions were displaying emotions that she had no control over, "my expressions are showing anger", and would hide her face in her hands or with her hair. She started checking her face in the mirror four to five times, and asking her mother for reassurance. She reported difficulty with memory and "my brain is going dead".

As a child, she was described as outgoing and social, but at age of 15 years, social anxiety presented, and at age of 18 years, she was seen by a psychologist to work on repetitive checking behavior, which she and her family reported resolved with brief treatment with venlafaxine. Although she did excellent in school and the first part of university, her grades began to drop and she switched universities, changing her original plan from studying medicine to nursing, and then kinesiology.

At age of 12 years, she had removal of a facial mole with 
Table 1. Testing done

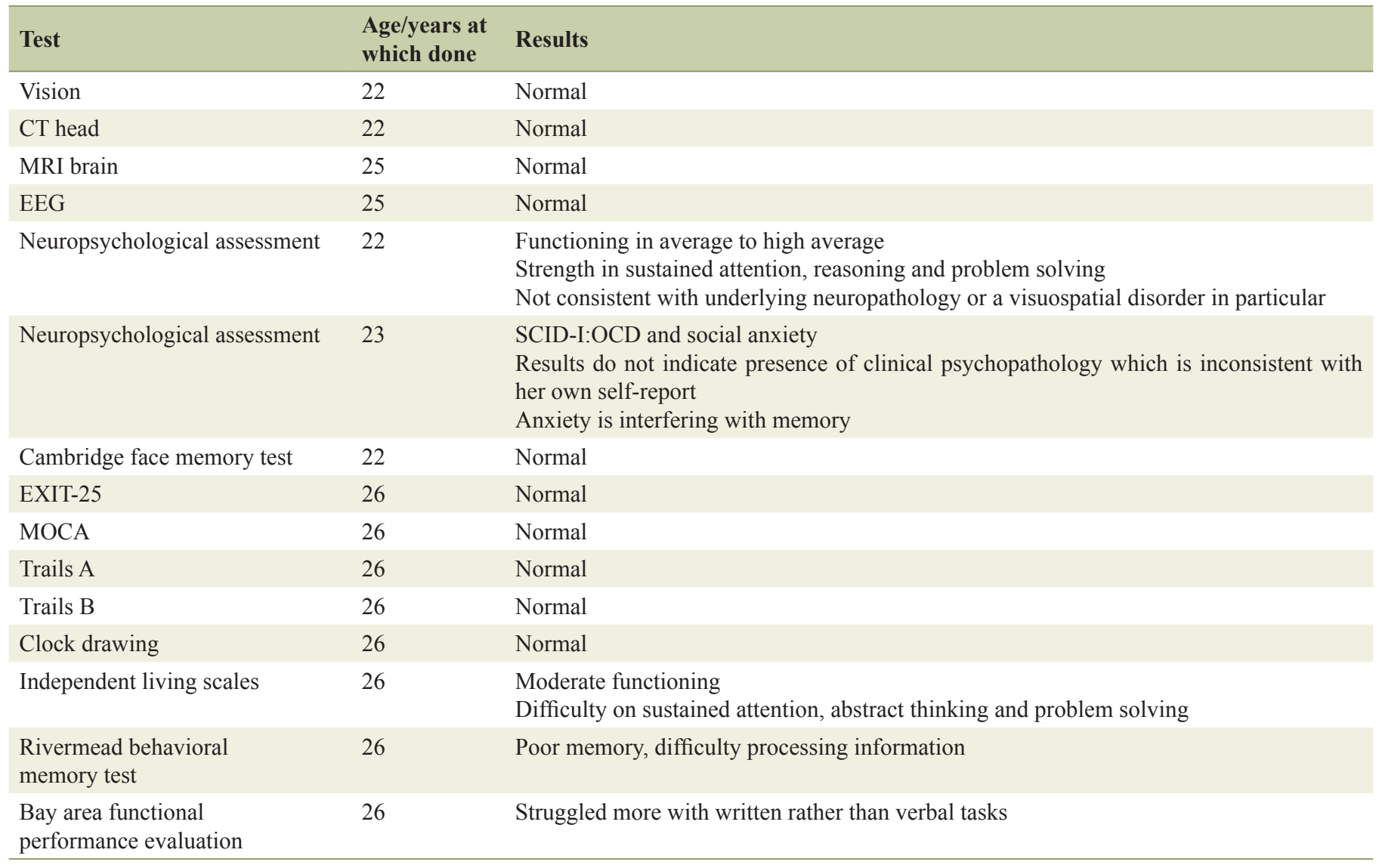

no complications, and at age of 18 years, she was diagnosed with Graves' disease, received radioiodine and was currently on eltroxin. Family history is positive for anxiety and Graves' disease in mother and depression in uncle and cousin, but no family history of bipolar disorder or schizophrenia.

Physical examination, eyesight, CT head, MRI brain, EEG and initial neuropsychological assessment including the Cambridge Face Memory test [12], were all reported as essentially normal, with pre-existing asthma, and hypothyroidism that was well controlled. At age of 22 years, IQ was noted as above average, although Ms. AB constantly reported that she had difficulty concentrating and remembering. On the neuropsychological testing, sustained attention was identified as a strength.

She was admitted to hospital four times, the second and third times due to attempted suicides because of fear and distress at the experiences. The fourth admission at age of 26 years was to manage clozapine and the experienced side effects. The first three admissions were at age 22 of years.

Neuropsychological testing was first conducted at age of 22 years, repeated at age of 23 years, and then at age of 26 years to help with community treatment planning (Table 1).

Differential diagnoses of anxiety, obsessive compulsive disorder, bipolar disorder and schizophrenia were discussed.

Over the years, as she moved to different towns to stay with different family members, she was seen in total by five different psychiatrists and two psychologists at different times.
The majority of these treatment providers, who all saw her in the first 6 months to a year from presentation, questioned the initial diagnosis of schizophrenia, leading to medications changes and repeated neuropsychological testing.

Risperidone, the first medication that was initially started, was reported by her as having some improvement in the "distortions"; however, she had two suicide attempts while on the medication and still continued to struggle. A series of medications, antidepressants (fluvoxamine, fluoxetine, venlafaxine), anticonvulsants (lamotrigine) and antipsychotics had limited benefit. Given the limited benefit from high doses of quetiapine, olanzapine, ziprasidone and clopixol, clozapine was discussed and the patient eventually agreed to it with reservations, and unfortunately had significant orthostatic hypotension, and white blood cell count dropped but was able to persist with support until benefit was noted.

At clozapine of $650-700 \mathrm{mg} /$ day, Ms. AB, for the first time, reported complete relief from the prosopometamorphosia. She had for the past 5 or so years held a steady job, and had lived in a group care home. Although functioning relatively well with the supports in place, she continued to report difficulties with concentration and presented with a flat affect.

In the last year, she reported a new onset of seeing "babies in the garbage". Clozapine was increased with limited effect, so aripriprazole was added. Ms. AB reported improvement and was back to what was her baseline functioning before the new 
visual hallucinations.

\section{Discussion}

Schizophrenia in females is known to be of later onset than in males [4]. Sometimes unusual first presentations such as prosopometamorphosia may confound the diagnosis.

Visual hallucinations in schizophrenia have been estimated to be about $27 \%$ versus auditory hallucinations being $59 \%$ in the same studies [5].

Visual hallucinations are more common in eye diseases and neurological conditions [6]. They are more common in younger individuals with schizophrenia, and have a bimodal presentation of onset, with the adolescence and young adulthood onset associated with psychosis [6] as noted with Ms. AB who was in her twenties.

Visual hallucinations rarely occur alone in schizophrenia, whereas auditory hallucinations commonly occur alone or in combination with visual hallucinations, but in organic disease, visual hallucinations predominate [6]. In psychosis, unlike organic conditions, the visual hallucinations usually have frightening content, emotional reactions and appraisals of personal significance, and cause distress as experienced by Ms. AB, leading to two suicide attempts.

There is limited evidence for specific pharmacological treatment for visual hallucinations in psychosis. Some studies report that visual hallucinations may be a marker for treatment resistance [6], and Ms. AB was trialed on many medications with limited benefit. Clozapine has been reported as effective for visual hallucinations [6], but studies on prosopometamorphasia in schizophrenia are lacking. Clozapine at $650-700$ $\mathrm{mg} /$ day was the first medication that completely controlled the prosopometamorphosia in Ms. AB.

The literature on organic diseases suggest the use of multiple different medications that might help prosopometamorphosia, including drugs that increase cholinergic activity, antidepressants, memantine anticonvulsants, but have not been examined for prosopometamorphosia in schizophrenia. None of the antidepressants used, most antipsychotics nor lamotrigine had benefit for the prosopometamorphosia for Ms. AB. except for clozapine.

On closer examination of the case, there are many indicators for a diagnosis of schizophrenia; however, the marked presenting positive symptom of prosopometamorphosia was striking as was the anxiety. She had experienced anxiety and deterioration in her academics, had blunted affect, was concerned about her expressions not being her own, and being controlled, and later in her life, endorsed the types of visual hallucinations that are more commonly described in schizophrenia.

\section{Conclusions}

Prosopometamorphosia is commonly associated with organic causes. Research into knowledge and management options in schizophrenia is warranted. This case illustrates the challenges faced when a presenting symptom is unusual and when medications are limited in efficacy.

\section{Acknowledgments}

We would like to acknowledge Dr. Lloyd Balbuena for his assistance.

\section{Conflict of Interest}

None.

\section{Financial Support}

None.

\section{References}

1. Blom JD. A Dictionary of Hallucinations. Sringer: New York, 2010.

2. Telles-Correia D, Moreira AL, Goncalves JS. Hallucinations and related concepts-their conceptual background. Front Psychol. 2015;6:991.

3. Ffytche DH, Blom JD, Catani M. Disorders of visual perception. J Neurol Neurosurg Psychiatry. 2010;81(11):1280-1287.

4. American Psychiatric Association. Diagnostic and statistical manual of mental disorders, Fifth Edition, 2013.

5. Teeple RC, Caplan JP, Stern TA. Visual hallucinations: differential diagnosis and treatment. Prim Care Companion J Clin Psychiatry. 2009;11(1):26-32.

6. Waters F, Collerton D, Ffytche DH, Jardri R, Pins D, Dudley R, Blom JD, et al. Visual hallucinations in the psychosis spectrum and comparative information from neurodegenerative disorders and eye disease. Schizophr Bull. 2014;40(Suppl 4):S233-245.

7. McCarty CW, Gordon GM, Walker A, Delio P, Kolarczyk RA, Pieramici DJ. Prosopometamorphopsia and alexia following left splenial corpus callosum infarction: Case report and literature review. eNeurologicalSci. 2017;6:1-3.

8. Barghouthi T, El Husseini N. Prosopometamorphopsia secondary to a left splenium of the corpus callosum infarct. BMJ Case Rep. 2018;2018.

9. Funatsu N, Hayakawa M, Tokuda N, Toyoda K. Transient Prosopometamorphopsia Restricted to the Left Eye Caused by Ischemia at the Right Splenium of the Corpus Callosum. Intern Med. 2017;56(21):2933-2935.

10. Norton JW, Corbett JJ. Visual perceptual abnormalities: hallucinations and illusions. Semin Neurol. 2000;20(1):111-121.

11. ffytche DH, Howard RJ. The perceptual consequences of visual loss: 'positive' pathologies of vision. Brain. 1999; 122(Pt 7):1247-1260.

12. Duchaine B, Nakayama K. The Cambridge Face Memory Test: results for neurologically intact individuals and an investigation of its validity using inverted face stimuli and prosopagnosic participants. Neuropsychologia. 2006;44(4):576-585. 